\title{
QUALIDADE DO SONO EM ESTUDANTES DO REGIME REGULAR E INTERNATO MÉDICO
}

\section{SLEEP QUALITY IN STUDENTS OF REGULAR GRADE AND MEDICAL INTERNS}

Gustavo Montibeller da Silva1, Fernando Arruda Ramos², Leonardo de Sousa Bernardes¹, Paulo Roberto Ramos Alves ${ }^{1}$, Matheus Antonio Fabro1

\section{RESUMO}

Objetivo: Esta pesquisa buscou conhecer o padrão de sono e a sonolência diurna dos estudantes de medicina no regime regular e no internato, bem como comparar os resultados obtidos. Metodologia: Realizou-se uma análise quantitativa transversal, com caráter descritivo, com os estudantes de medicina da Universidade do Planalto Catarinense. Foram aplicados questionários que continham os instrumentos Pittsburgh Sleep Quality Index(PSQI) para avaliação objetiva da qualidade do sono e o Epworth Sleepiness Scale(ESS) para avaliação da sonolência diurna. Os acadêmicos foram divididos em dois grupos sendo o primeiro do $1-4^{\circ}$ ano com turno regular e os estudantes do 5- $6^{\circ}$ ano no regime de internato. E após foram comparados estatisticamente. Resultados: Participaram da pesquisa 134 estudantes, sendo 60 homens (44,8\%) e 74 mulheres (55,2\%), com média de idade de 24,05 anos (DP $\$ 3,66$ ).Quanto aos resultados dos questionários foi verificada uma média do índice total do PSQI de 6,48 pontos ( $\mathrm{DP} \pm 4,71$ ) e da ESS 9,30 pontos ( $\mathrm{DP} \pm 4,71$ ). Os estudantes do internato apresentaram menor duração do sono, pior sensação subjetiva da qualidade do sono, enquanto os acadêmicos do turno regular apresentaram maior latência do sono $p<0,05$. Não houve diferenças estatísticas entre os grupos dos escores totais do PSQI e ESS, sendo que, $59 \%$ possuem qualidade ruim de sono. Conclusão: É notável a má qualidade do sono, com importante diminuição das horas totais dormidas. As implicações da insônia crônica e sua influência no desempenho acadêmico e profissional já estão bem estabelecidas, há necessidade de intervenção nas grades curriculares, medidas indispensáveis para correção deste problema.

Descritores: Estudantes de Medicina. Sono. Privação do Sono. Distúrbios do Sono por Sonolência Excessiva. Transtornos do Sono.

\section{ABSTRACT}

Objective: This research aimed to know the pattern of sleep and daytime sleepiness of medical students in regular grade and medical internsand compare the results. Methodology: We performed a cross-sectional quantitative analysis, with descriptive character, with medical students at the University of Planalto Catarinense. Questionnaires were applied containing the Pittsburgh Sleep Quality Index to objectively evaluate quality of sleep and the Epworth Sleepiness Scale for assessing daytime sleepiness. The students were divided into two groups being the first of the 1-4th year with regular shift and the medical internson the 5-6th year. And after were compared statistically. Results: Participated in the study 134 students, 60 men $(44.8 \%)$ and 74 women (55.2\%) with mean age of 24.05 years (SD \pm 3.66$)$. As the measures the questionnaires found a mean total PSQI index of 6.48 points $(S D \pm 4.71$ ) and ESS 9.30 points $(S D \pm 4.71)$. Medical interns had shorter duration of sleep, feeling worse subjective sleep quality, while the students of the regular grade had greater sleep latency $p<0.05$. There no statistical differences between the groups of the total scores of PSQI and ESS, being that 59\% have poor quality sleep. Conclusion: It is notable the poor sleep quality, with a significant reduction of total hours of slept. The implications of chronic insomnia and its influence on academic and professional performance are already well established, there is need for intervention in curricular grade, indispensable measures to correct this problem.

Keywords: Students, Medical. Sleep. Sleep Deprivation. Disorders of Excessive Somnolence. Sleep Disorders.

1. Acadêmico do curso de Medicina da Universidade do Planalto Catarinense

2. Médico otorrinolaringologista, Mestre em Ciências Médicas, docente do curso de medicina da Universidade do Planalto Catarinense

Contato do Autor / Mail to:

Gustavo Montibeller da Silva - gustavo.montibeller@hotmail.com

Rua Prudente de Morais, 958, ap13, Lages-SC. CEP 88523-135

Fone: (49) 9954-9394 


\section{INTRODUÇÃO}

O sono desempenha papel vital na saúde, desde a antiguidade, o processo do sono gera curiosidade nos homens. Aristóteles acreditava que o sono era necessário para manter a percepção, que caso utilizada ininterruptamente se esgotaria. Hipócrates já associava a insônia ao aborrecimento e à tristeza, uma de suas celebres frases dizia "Se o sono é um ensaio para a morte, acordar é um ensaio para a vida”, apesar de todo o interesse filosófico somente na segunda metade do século XX o sono passou a interessar cientificamente ${ }^{1,2}$.

A privação de sono é um problema crônico das sociedades modernas, ela aparece como uma importante causa de baixa imunidade, elevando o risco a longo prazo para desenvolvimento de doenças cardiovasculares, diabetes, e doenças neuro degenerativas como a doença de Alzheimer e esclerose múltipla ${ }^{3}$.

Processos celulares e moleculares na área do hipocampo que ocorrem durante o sono NREM são essenciais no processo de plasticidade cerebra|3,4, que por sua vez estão subjacentes a aprendizagem e memória. Indicações que sono participa na consolidação de memória recente vêm de uma ampla gama de estudos experimentais e observacionais 5 .

Noites de sono mal dormidas traduzem-se em prejuízos cognitivos como diminuição da capacidade de fixar e manter a atenção, perda da capacidade de planejamento estratégico, prejuízo da memória, prejuízos motores, impulsividade e raciocínio deteriorado, aumento do cortisol e diminuição na imunidade, resultando em prejuízos nos estudos, no trabalho, nas relações familiares e sociais6.

Resultados consistentes sugerem que a insônia crônica é um marcador para indivíduos sob risco de desenvolverem doenças psiquiátricas. Resultados de trabalhos mostram que a insônia crônica confere um risco acumulado para o desenvolvimento de ansiedade e depressão em adultos saudáveis bem como pode ser uma manifestação precoce dessas doenças ${ }^{7}$.

Estudantes de medicina representam um grupo socialmente predisposto a alterações no ciclo sono vigília. Mudanças ambientais, sociais e compromissos curriculares, limitam a qualidade do sono, pois os estudantes acabam tendo que decidir entre manter a qualidade do sono, satisfazendo suas necessidades, ou responder aos esquemas acadêmicos e curriculares ${ }^{8}$. A alta cobrança a respeito dos estudantes de medicina, por sua carga horária e compromissos curriculares afetam diretamente a qualidade do sono. Por natureza precisamos de regularidade no padrão sono e vigília, estudantes universitários devido a afazeres acadêmicos tendem a reduzir a quantidade total de horas dormidas.

Os quatro primeiros anos da faculdade concentram em grande parte as áreas teóricas do curso enquanto que o Internato Médico passa a realizar o atendimento direto, com uma carga horária maior e por vezes com regime de plantões, para passar o máximo da vivencia médica para os acadêmicos. Sendo assim, este estudo foi desenvolvido na expectativa de conhecer o padrão do sono de internos e estudantes de turno regular bem como comparar se existe diferença estatística entre a qualidade e distribuição do sono e sonolência diurna destes estudantes.

\section{METODOLOGIA}

Este estudo trata de uma análise quantitativa transversal, com caráter descritivo. Tem por objetivo conhecer o padrão de sono, e a sonolência diurna dos estudantes de medicina no regime regular e no internato e comparar os resultados obtidos entre os dois grupos.

A pesquisa foi realizada na Universidade do Planalto Catarinense (UNIPLAC) no período de 5 de abril de 2015 até 29 de novembro de 2015, o período de recrutamento foi de 5 de novembro até 29 de novembro de 2015. Participaram do estudo maiores de 18 anos, que concordaram em participar da pesquisa, foram recrutados estudantes do $1^{\circ}$ ao $6^{\circ}$ ano do curso de medicina da UNIPLAC, escolhidos ao acaso, de um total de 245 alunos regularmente matriculados no curso.

0 instrumento de coleta de dados consiste de dois questionários já validados na língua portuguesa. 0 Pittsburgh Sleep Quality Index (PSQI), que é um questionário autoaplicável, que aborda a qualidade e os distúrbios apresentados do sono no intervalo de um mês avaliando-os qualitativa e quantitativamente. Possui dez questões, tendo 19 itens individuais que geram um escore composto de sete itens entre eles, a qualidade subjetiva do sono, latência do sono, duração do sono, eficiência habitual do sono, distúrbios do sono, uso de medicações para dormir e a disfunção diurna. Cada item gera um valor que varia 0 (melhor) a 3 (Pior) a soma individual desses critérios formam um escore global que varia de 0 a 21 pontos, sendo que pontuações maiores que 5 indicam qualidade ruim de sono que com $89,6 \%$ de sensibilidade e $86,5 \%$ de especificidade ${ }^{9-11}$.

Outro instrumento utilizado foi a Epworth Sleepiness Scale (ESS) ${ }^{12}$, ele avalia a sonolência diurna, sendo um questionário auto administrado, referindo à possibilidade de cochilar durante atividades cotidianas. Com oito questões utilizando-se uma escala de 0 a 3 para cada uma delas, onde zero é a mínima possibilidade de cochilar e três é grande possibilidade de cochilar. A soma das pontuações individuais forma um escore global, sendo que escores maiores que 10 são indicativos sonolência diurna excessiva ${ }^{10,13 .}$

Para compreender os resultados os acadêmicos foram divididos em dois grupos sendo o primeiro do $1-4^{\circ}$ ano com turno regular e os estudantes do $5-6^{\circ}$ ano no regime de internato. $\mathrm{Na}$ expectativa de evitar vieses os estudantes foram abordados em grupos, e as datas de coletas, tinham ao menos 5 dias anteriores ou posteriores a datas de provas. 
Os dados foram computados no software Microsoft $\circledast$ Acess 2016, tabulados no Microsoft ${ }^{\circledR}$ Excel 2016 e analisados no IBM ${ }^{\circledR}$ SPSS 22. Para tratamento dos dados as análises de média e desvio padrão para informações da população, o teste de Qui-quadrado para analises nominais e T-Student mostra independentes para analises nominais/ordinais considerando estatisticamente significantes quando $\mathrm{p}$ menor que 0,05 com intervalo de confiança de $95 \%$.

A pesquisa foi devidamente aprovada no comitê de ética em pesquisa pelo relatório CAEE 30569814.5.0000.5368.

\section{RESULTADOS}

Participaram da pesquisa 141 estudantes, destes 7 foram excluídos pois não assinaram o TCLE ou não preencherem adequadamente os instrumentos, totalizando ao final 134 participantes, sendo 60 homens (44,8\%) e 74 mulheres $(55,2 \%)$, com média de idade de 24,05 anos $(\mathrm{DP} \pm 3,66)$.

Participaram por ano de graduação do primeiro ano 27 alunos $(20,1 \%)$, do segundo ano 19 alunos $(14,2 \%)$, do terceiro ano 22 alunos $(16,4 \%)$, do quarto ano 29 alunos (21,6\%), do quinto ano 18 alunos $(13,4 \%)$ e do sexto ano 19 alunos (14,2\%), na divisão de grupos 97 $(72,4 \%)$ participam do regime regular e $37(27,6 \%)$ são estudantes do internato (Tabela 1).

Tabela 1. Características da população estudada

\begin{tabular}{lll} 
Gênero & $\mathrm{N}$ & $\%$ \\
\hline Masculino & 60 & 44,8 \\
\hline Feminino & 74 & 55,2 \\
\hline Ano & $\mathrm{N}$ & $\%$ \\
\hline $1^{\circ}$ & 27 & 20,1 \\
\hline $2^{\circ}$ & 19 & 14,2 \\
\hline $3^{\circ}$ & 22 & 16,4 \\
\hline $4^{\circ}$ & 29 & 21,6 \\
\hline $5^{\circ}$ & 18 & 13,4 \\
\hline $6^{\circ}$ & 19 & 14,2 \\
\hline
\end{tabular}

Quanto as medidas dos questionários encontramos uma média do índice total do PSQI de 6,48 pontos $(\mathrm{DP} \pm 4,71)$ e da ESS 9,30 pontos (DP $\pm 4,71)$. 0 Tempo médio para iniciar o sono foi de $27,33 \mathrm{~min}$ (DP $\pm 21,36$ ), a hora média de acordar foi de $7 \mathrm{~h} 37 \mathrm{~min}$ (DP $\pm 1 \mathrm{~h} 12 \mathrm{~min}$ ), o total de horas dormidas por noite foi de $6,66 h(D P \pm 1,28 h)$.
Quando analisados individualmente 22 classificaram a qualidade do sono como muito boa $(16,4 \%), 81$ como boa $(60,4 \%), 30$ como ruim $(22,4 \%)$ e um como Muito Ruim (0,7\%). Quando comparados estudantes do regime regular em relação aos estudantes do internato existe diferença significativa $(p=0,04)$.

Em relação ao uso de medicação hipnótica 84,3\% não fazem uso de nenhuma medicação, e cerca de 15,7\% fazem uso desse tipo de recurso para induzir o sono sendo que não há diferença estatística entre regime de regular e internato $(p=0,393)$, e entre o sexo masculino e feminino $(p=0,521)$.

Quanto a duração do sono no regime regular a duração do sono ficou em 7,02h ( $D P \pm 1,22)$, e no grupo do internato $5,73 \mathrm{~h}(\mathrm{DP} \pm 0,87)$, havendo diferença significativa entre os dois grupos. E para valores somatórios a média de pontos foi de $0,48(\mathrm{DP} \pm 0,72)$ no regime regular e 1,30 $(\mathrm{DP} \pm 0,87)$ no regime de internato, com valor de p 0,001.

Nos distúrbios do sono a média em pontos para o grupo em regime regular foi de 1,24 $(\mathrm{DP} \pm 0,51)$ e 1,27 para o grupo em regime de internato $(\mathrm{DP} \pm 0,65)$, sem diferença estatística entre os grupos.

Quando analisados a latência do sono no grupo em regime regular existe um valor maior em pontos quando comparados com o grupo em regime de internato, de respectivamente, 1,71 (DP $\pm 0,91)$ e 1,19 (DP $\pm 0,96)$ estatisticamente significantes.

Em relação a disfunção diurna os grupos apresentam no regime normal e internato respectivamente em pontos 1,28 $(\mathrm{DP} \pm 0,80)$ e 1,22 (DP $\pm 0,75)$, sem diferenças estatísticas entre os grupos $p>0,05$. $\mathrm{Na}$ questão de eficiência do sono não existe relação de significância estatística entre grupos no regime regular $0,39(\mathrm{DP} \pm 0,84)$ e internato 0,35 (DP $\pm 0,88)$

De acordo com a soma total do PQSI encontramos, $41 \%$ (55 alunos) possuem boa qualidade do sono e 59\% (79 alunos) possuem qualidade de sono ruim, não há diferenças estatísticas significativas $p>0,05$, entre estudantes do turno regular $6,36(\mathrm{DP} \pm 3,00)$ e internato $6,78(\mathrm{DP} \pm 0,2,41)$ (Tabela 2).

No questionário ESS 100 (74,6\%) apresentavam sonolência diurna excessiva e apenas $34(25,4 \%)$ tinha este índice normal. Quando comparados os grupos não há significância estatística $(p=0,24)$.

Quando comparados aos homens as mulheres participantes deste estudo apresentam uma maior duração do Sono $(P=0,010)$, mais distúrbios do sono $(p=0,33)$ e maior disfunção diurna $(p=0,001)$, além de apresentarem uma maior sonolência diurna $(p=0,001)$. Eficiência do sono, latência do sono, uso de medicações, qualidade subjetiva do sono e o escore global do PSQI apresentam-se semelhantes entre os gêneros (Tabela 3). 


\section{DISCUSSÃO}

Como já evidenciado é clara a importância de se manter uma boa qualidade do sono, a insônia além de implicar em prejuízos de memória e concentração, leva a um quadro crônico de baixo desempenho afetivo e acadêmico.

Em relação aos estudantes é notável a má qualidade do sono, com importante diminuição das horas totais de sono com apenas $6,6 \mathrm{~h}$ diárias semelhantes a estudos anteriores 2 , 14 que são muito abaixo do recomendado para adultos de $8 \mathrm{~h}$ de sono diárias ${ }^{15}$. 0 tempo para iniciar o sono de 27,3 minutos muito próximo de valores de 30 minutos adotados para caracterizar insônia crônica16, estes associados a um despertar por volta das $7 \mathrm{~h} 37 \mathrm{~min}$.Em contraste com os resultados do PSQI cerca de $76,8 \%$ classificaram sua qualidade subjetiva do sono como boa ou muito boa, apesar dos resultados alarmantes evidenciados onde 59\% apresentam-se com qualidade do sono ruim.

Quando comparamos a percepção da qualidade do sono, há uma notável redução da percepção da qualidade do sono, onde mais estudantes do internato passam a classificar o sono como ruim, associado a está a diminuição da quantidade de horas dormidas que passam de $7,02 \mathrm{~h}$ para $5,73 \mathrm{~h}$ quando comparados internato e ensino regular, um valor muito abaixo das necessidades diárias, a redução das horas de sono associados a regimes de plantão, são problemas crônicos conhecidos de médicos e residentes, estes que se apresentam com um custo social enorme que tornam incompativeis com um sistema de saúde seguro e efetivo, tal fato já evidenciado por Gaba e Howard em 200217. Apesar de tal fato ser conhecido de profissionais, a origem deste problema pode ser evidenciada já durante a faculdade.

0 uso de medicações hipnóticas foi outro dado preocupante onde $15,7 \%$ do total de estudantes admitem usar medicações para induzir o sono, valor que é muito superior a estudos populacionais Brasileiro que evidenciam a pratica em 6,9\%18 em campo grande e 3,3\% nas 107 cidades brasileiras com mais de 200mil habitantes ${ }^{19}$. Respectivamente não existe diferença estatística entre internato e ensino regular, $p=0,393$., quanto ao uso destas medicações, bem como diferenças entre o sexo masculino e feminino.

A latência do sono no PSQI é composta da soma dos valores gerados pelo tempo para iniciar o sono mais a frequência de vezes na semana que levou mais de 30 minutos para dormir. Neste caso os estudantes do internato apresentam menor latência para iniciar o sono quando comparados a estudantes do turno regular, o aumento da latência para iniciar o sono é fortemente correlacionado com distúrbios de depressão e ansiedade ${ }^{20}$ 22. Outras relações mensuradas pelo PSQI como: distúrbios do sono, disfunção diurna e eficiência do sono apresentam resultados semelhantes para os dois grupos.

A fadiga crônica é um problema já conhecido de vivida por médicos e residentes de medicina, problema em grande parte devido à carga horária com mais de $80 \mathrm{~h} /$ semanais e plantões regulares de $24 \mathrm{~h}$ ou mais ${ }^{17}$, a cidade de Nova lorque foi pioneira ao regulamentar os horários de médicos residentes, e aumentar a supervisão sobre estes, para evitar problemas relacionados com a carga de trabalho excessiva23, ao analisar podemos relacionar os problemas apresentados por médicos e residentes como uma extensão do que ocorre com os acadêmicos de medicina, altas carga horárias semanais, regimes de plantões e horários incertos, que traduzem-se

Tabela 2. Distribuição das variáveis do PSQI, escore total e escore total do ESS entre estudantes do Ensino Regular e Internato

\begin{tabular}{llll}
\hline Variável & Ensino Regular & Internato & $\mathrm{P}$ \\
\hline & $\mathrm{N}=97$ & $\mathrm{~N}=37$ & \\
\hline Qualidade Subjetiva do Sono & $0,99 \pm 0,63$ & $1,3 \pm 0,61$ & 0,013 \\
\hline Latência do Sono & $1,71 \pm 0,91$ & $1,19 \pm 0,96$ & 0,004 \\
\hline Duração do Sono & $0,48 \pm 0,72$ & $1,3 \pm 0,87$ & 0,001 \\
\hline Eficiência do Sono & $0,39 \pm 0,84$ & $0,35 \pm 0,88$ & 0,808 \\
\hline Distúrbios do Sono & $1,24 \pm 0,51$ & $1,27 \pm 0,65$ & 0,758 \\
\hline Uso de Medicações & $0,27 \pm 0,70$ & $0,16 \pm 0,44$ & 0,393 \\
\hline Disfunção Diurna & $1,28 \pm 0,80$ & $1,22 \pm 0,75$ & 0,683 \\
\hline PSQI & $6,36 \pm 3,0$ & $6,78 \pm 2,41$ & 0,444 \\
\hline ESS & $9,57 \pm 4,70$ & $8,59 \pm 4,71$ & 0,282 \\
\hline
\end{tabular}


Tabela 3. Distribuição das variáveis do PSQI, escore total e escore total do ESS entre homens e mulheres

\begin{tabular}{|c|c|c|c|}
\hline Variável & Homens & Mulheres & $\mathbf{P}$ \\
\hline & $N=60$ & $N=74$ & \\
\hline Qualidade & $1,05 \pm 0,69$ & $1,09 \pm 0,60$ & 0,692 \\
\hline \multicolumn{4}{|l|}{ Subjetiva do Sono } \\
\hline Latência do Sono & $1,58 \pm 0,94$ & $1,55 \pm 0,96$ & 0,860 \\
\hline Duração do Sono & $0,92 \pm 0,90$ & $0,54 \pm 0,76$ & 0,010 \\
\hline Eficiência do & $0,27 \pm 0,73$ & $0,74 \pm 0,94$ & 0,166 \\
\hline \multicolumn{4}{|l|}{ Sono } \\
\hline Distúrbios do & $1,13 \pm 0,56$ & $1,34 \pm 0,53$ & 0,033 \\
\hline \multicolumn{4}{|l|}{ Sono } \\
\hline Uso de & $0,22 \pm 0,58$ & $0,26 \pm 0,68$ & 0,720 \\
\hline \multicolumn{4}{|l|}{ Medicações } \\
\hline Disfunção Diurna & $1,02 \pm 0,65$ & $1,46 \pm 0,83$ & 0,001 \\
\hline PSQI & $6,18 \pm 3,02$ & $6,72 \pm 2,69$ & 0,283 \\
\hline ESS & $7,8 \pm 3,94$ & $10,5 \pm 4,94$ & 0,001 \\
\hline
\end{tabular}

em perda da qualidade do sono, mostrando que a rotina do interno médico em muito assemelha-se com os profissionais já graduados.

0 índice total do PSQI demonstrou outro grande problema onde o escore total foi de 6,48 pontos, valores que caracterizam má qualidade do sono, enquanto na população em geral que compões o grupo controle de um estudo do Hospital das Clínicas de Porto Alegre, a população obteve 2,5 pontos $^{24}$ enquanto no estudo original esses valores foram de 2,64 pontos para a população em geral9.

Quanto ao ESS apresentou resultado igualmente preocupante, onde encontrou-se uma média 9,30 pontos $(\mathrm{DP} \pm 4,71)$, sendo que $74 \%$ apresentavam sonolência diurna excessiva, valores muito superiores aos encontrados em estudos anteriores com outros estudantes de cursos da saúde $50 \% 25$ estudantes de medicina $44,7 \%^{2}$ e $34,6 \% 14$.

Mulheres são mais propensas a relatarem mais sintomas de insônia, apresentam maior sonolência diurna e insatisfação com o sono26, neste estudo pode se evidenciar pelo PSQI, que mesmo tendo uma quantidade maior de horas dormidas por noite as mulheres tendem a terem mais distúrbios do sono, disfunção diurna pelo sono perdido bem como pela avaliação do ESS apresentam uma maior sonolência diurna, correlacionando com resultados de estudos anteriores 27,28 .

\section{CONCLUSÃO}

Estudantes de medicina compõem um grupo especial de risco para desenvolvimento de distúrbios do sono, é notável a diminuição do total de horas dormidas, aumento da latência do sono e consequente aumento da sonolência diurna, além destes há um grande consumo de drogas hipnóticas pelos estudantes.

Apesar da sensação subjetiva de boa qualidade do sono, os estudantes acabam recebendo pontuações altas no PSQI, que é um questionário altamente validado com boa sensibilidade e especificidade.

Assim comparados estudantes do turno regular e estudantes do internato, é notável a diminuição da quantidade de horas dormidas, bem como a percepção subjetiva da qualidade do sono, porém quando analisados na totalidade os estudantes apresentam qualidade de sono semelhantemente ruins.

As implicações da insônia crônica e sua influência no desempenho acadêmico e profissional já estão bem estabelecidas, a alta prevalência de má qualidade de sono e os índices de sonolência diurna excessiva nos estudantes de medicina preocupam, sendo que há a necessidade de gestores e docentes intervenção no planejamento curricular de um curso de medicina, para que as atividades sejam distribuídas e respeitem as necessidades de sono dos estudantes. Para tal é 
necessário conhecer o perfil destes estudantes e do currículo proposto. Quando visados essas alterações são indispensáveis para correção deste problema grave e crônico de médicos, mas que tem origem ainda na universidade.

\section{CONFLITOS DE INTERESSE}

Os autores deste artigo não possuem nenhum conflito de interesse.

\section{REFERÊNCIAS}

1. Drabovicz PV, Salles V, Drabovicz PE, Fontes MJ. Avaliação da qualidade do sono em adolescentes com disfunções temporomandibulares. J pediatr(Rio J). 2012;88(2):169-72.

2. Cardoso HC, Bueno FCdC, Mata JCd, Alves APR, Jochims I, Vaz Filho IHR, et al. Assessing quality of sleep in medical students. Revista Brasileira de Educação Médica. 2009;33(3):349-55.

3. Prince TM, Abel T. The impact of sleep loss on hippocampal function. Learn Mem. 2013 Oct;20(10):558-69. PubMed PMID: 24045505. Pubmed Central PMCID: 3768199.

4. Rocha CR, Rossini S, Reimão R. Sleep disorders in high school and pre-university students. Arquivos de neuro-psiquiatria. 2010;68(6):903-7.

5. Maquet $P$. The role of sleep in learning and memory. science. 2001;294(5544):1048-52.

6. Müller MR, Guimarães SS. Impacto dos transtornos do sono sobre o funcionamento diário e a qualidade de vida. Estudos de Psicologia (Campinas). 2007;24:51928.

7. Neckelmann D, Mykletun A, Dahl AA. Chronic insomnia as a risk factor for developing anxiety and depression. SLEEP-NEW YORK THEN WESTCHESTER-. 2007;30(7):873.

8. Martini ${ }^{1}$ M, Brandalize M, Louzada FM, Pereira ÉF, Brandalize D. Fatores associados à qualidade do sono em estudantes de Fisioterapia. 2012.

9. Buysse DJ, Reynolds CF, 3rd, Monk TH, Berman SR, Kupfer DJ. The Pittsburgh Sleep Quality Index: a new instrument for psychiatric practice and research. Psychiatry Res. 1989 May;28(2):193-213. PubMed PMID: 2748771. Epub 1989/05/01. eng.

10. Togeiro SMGP, Smith AK. Métodos diagnósticos nos distúrbios do sono Diagnostics methods for sleep disorders. Rev Bras Psiquiatr. 2005;27(Supl I):8-15.

11. Omachi TA. Measures of sleep in rheumatologic diseases: Epworth Sleepiness Scale (ESS), Functional Outcome of Sleep Questionnaire (FOSQ), Insomnia Severity Index (ISI), and Pittsburgh Sleep Quality Index (PSQI). Arthritis Care Res (Hoboken). 2011 Nov;63 Suppl 11:S287-96. PubMed PMID: 22588751. Pubmed Central PMCID: Pmc3711174. Epub 2012/05/25. eng.

12. Johns MW. A new method for measuring daytime sleepiness: the Epworth sleepiness scale. Sleep. 1991
Dec;14(6):540-5. PubMed PMID: 1798888. Epub 1991/12/01. eng.

13. Bertolazi AN. Tradução, adaptação cultural e validação de dois instrumentos de avaliação do sono: Escala de Sonolência de Epworth e Índice de Qualidade de Sono de Pittsburgh. 2008.

14. Ribeiro CRF, da Silva YMGP, de Oliveira SMC. O impacto da qualidade do sono na formação médica. Rev Soc Bras Clín Méd. 2014;12(1).

15. Verster JC, Pandi-Perumal SR, Streiner DL. Sleep and quality of life in clinical medicine: Springer; 2008.

16. Silber MH. Chronic insomnia. New England Journal of Medicine. 2005;353(8):803-10.

17. Gaba DM, Howard SK. Fatigue among clinicians and the safety of patients. New England Journal of Medicine. 2002;347(16):1249-55.

18. Souza JC, Magna LA, Reimão R. Insomnia and hypnotic use in Campo Grande general population, Brazil. Arquivos de Neuro-Psiquiatria. 2002;60:702-7.

19. Carlini E, Galduróz JCF, Noto AR, Nappo SA. I Levantamento domiciliar sobre o uso de drogas psicotrópicas no Brasil: estudo envolvendo as 107 maiores cidades do país. São Paulo: Cebrid/Unifesp. 2002.

20. Staner L. Sleep and anxiety disorders. Dialogues Clin Neurosci. 2003 Sep;5(3):249-58. PubMed PMID: 22033804. Pubmed Central PMCID: Pmc3181635. eng.

21. Lakshminarayana Tadimeti M, Caruana-Montaldo B, Wallace B, Mendelson M. Sleep latency and duration estimates among sleep disorder patients: variability as a function of sleep disorder diagnosis, sleep history, and psychological characteristics. Sleep. 2000;23(1):1.

22. Reynolds CF, Shaw DH, Newton TF, Coble PA, Kupfer DJ. EEG sleep in outpatients with generalized anxiety: a preliminary comparison with depressed outpatients. Psychiatry research. 1983;8(2):81-9.

23. Wallack MK, Chao L. Resident work hours: the evolution of a revolution. Archives of surgery. 2001;136(12):1426-32.

24. Bertolazi AN, Fagondes SC, Hoff LS, Dartora EG, Miozzo IC, de Barba ME, et al. Validation of the Brazilian Portuguese version of the Pittsburgh Sleep Quality Index. Sleep Med. 2011 Jan;12(1):70-5. PubMed PMID: 21145786. Epub 2010/12/15. eng.

25. Pascotto AC, dos Santos BRM. Avaliação da qualidade do sono em estudantes de ciências da saúde.

26. Ohayon MM. Epidemiology of insomnia: what we know and what we still need to learn. Sleep medicine reviews. 2002;6(2):97-111.

27. Hetta J, Broman J, Mallon L. Evaluation of severe insomnia in the general population-implications for the management of insomnia: insomnia, quality of life and healthcare consumption in Sweden. J Psychopharmacol. 1999;13:S35.

28. Doi $Y$, Minowa M, Okawa M, Uchiyama M. Prevalence of sleep disturbance and hypnotic medication use in relation to sociodemographic factors in the general Japanese adult population. Journal of epidemiology. 2000;10(2):79-86. 